\title{
Face, content, and construct validity of human placenta as a haptic training tool in neurointerventional surgery
}

\author{
Marcelo Magaldi Ribeiro de Oliveira, MD, PhD, ${ }^{1-3}$ Arthur Nicolato, MD, ${ }^{1}$ Marcilea Santos, MD, ${ }^{1}$ \\ Joao Victor Godinho, MD, ${ }^{1}$ Rafael Brito, MD, ${ }^{1}$ Alexandre Alvarenga, MD, ${ }^{1}$ \\ Ana Luiza Valle Martins, MD, ${ }^{1}$ André Prosdocimi, MD, ${ }^{1}$ Felipe Padovani Trivelato, MD, ${ }^{1}$ \\ Abdulrahman J. Sabbagh, MBBS, FRCSC, ${ }^{4}$ Augusto Barbosa Reis, MD, PhD, and \\ Rolando Del Maestro, MD, PhD²
}

'Department of Surgery, Federal University of Minas Gerais, Brazil; ${ }^{2}$ Department of Neurosurgery, Neurosurgical Simulation Research and Training Centre, Montreal Neurological Institute and Hospital, McGill University, Montreal, Quebec; ${ }^{3}$ Surgical Simulation Centre, Mount Sinai Hospital, University of Toronto, Ontario, Canada; and ${ }^{4}$ Department of Neurosurgery, National Neurosciences Institute, King Fahad Medical City, Riyadh, Saudi Arabia

\begin{abstract}
OBJECTIVE The development of neurointerventional treatments of central nervous system disorders has resulted in the need for adequate training environments for novice interventionalists. Virtual simulators offer anatomical definition but lack adequate tactile feedback. Animal models, which provide more lifelike training, require an appropriate infrastructure base. The authors describe a training model for neurointerventional procedures using the human placenta (HP), which affords haptic training with significantly fewer resource requirements, and discuss its validation.

METHODS Twelve HPs were prepared for simulated endovascular procedures. Training exercises performed by interventional neuroradiologists and novice fellows were placental angiography, stent placement, aneurysm coiling, and intravascular liquid embolic agent injection.
\end{abstract}

RESULTS The endovascular training exercises proposed can be easily reproduced in the HP. Face, content, and construct validity were assessed by 6 neurointerventional radiologists and 6 novice fellows in interventional radiology.

CONCLUSIONS The use of HP provides an inexpensive training model for the training of neurointerventionalists. Preliminary validation results show that this simulation model has face and content validity and has demonstrated construct validity for the interventions assessed in this study.

http://thejns.org/doi/abs/10.3171/2015.1.JNS141583

KEY WORDS placenta; interventional neuroradiology; training model; surgical simulation validation; vascular disorders

$\mathrm{N}$ EUROINTERVENTIONAL procedures have become a significant asset in the treatment of cerebrovascular disorders. Training in these techniques requires several years of dedicated study to develop an understanding of and the haptic feel for catheter navigation and interventional treatments. However, medicolegal concerns and work hour restrictions may limit the experience trainees receive. Simulators allow trainees to improve their technical expertise and also allow physicians and industry to collaborate in the development of innovative devices. ${ }^{3}$
The ideal training model should be inexpensive, readily available, and have haptic characteristics similar to those encountered in the endovascular treatment of human disorders. Animal and computer-based models have been developed for this purpose. ${ }^{3,5,7}$ While each model has certain advantages and disadvantages, it is difficult to reproduce all the haptic qualities necessary for these procedures using virtual simulators or animal models. ${ }^{4,6-8}$ Thus, it is necessary to continue to develop and research new techniques for neurointerventional training. In this article, we 
describe and discuss the validation of a new, inexpensive, biological, and readily available training model using a human placenta (HP) - a model that can be reproduced in most teaching institutions.

\section{Methods}

Following ethics committee approval at the Federal University of Minas Gerais, Brazil, in 2011, HPs were donated following parturition and after informed consent, specifically for practice in surgical techniques. The specimens were registered with the pathology department and labeled with any relevant infection status of the donor to minimize biological contamination upon collection. Different types of aneurysms were created in the HPs using techniques described by the first author (M.M.R.d.O.) and colleagues in a previous publication. ${ }^{12}$

Twelve HPs were cleaned and prepared for use in the angiography suite (Toshiba Medical Systems). The following materials were required for this experiment: an infusion pump, a No. 6 urinary catheter, and a nonionic lowosmolar contrast agent (Optiray 300 [ioversol injection], Covidien). Also required was the full complement of tools necessary for neurointerventional treatments including: a 6-Fr guiding catheter (Guider Soft Tip, Stryker Neurovascular); microcatheters (Excelsior SL10, Stryker Neurovascular; Rebar 18, ev3; and Marathon, ev3); microguidewires (Transend EX, Stryker Neurovascular; and Mirage, ev3); a self-expanding stent (Solitaire, ev3); a liquid embolic agent (Onyx Liquid Embolic System, ev3); and Guglielmi detachable coils (GDCs, Stryker Neurovascular).

The authors prepared 12 HPs, clearing all vessels of blood clots and continuously infusing saline; a small aneurysm was created in an HP branch vessel, and an incision was made in 1 small vessel to simulate iatrogenic damage to the artery.

All of the procedure simulation exercises were performed in the HPs by the 12 participants. Six of the participants were considered to be "experts," defined in this research protocol as having performed at least 4 therapeutic endovascular procedures per month in the last 2 years. These participants work as interventional neuroradiologists in 3 different hospitals in the state of Minas Gerais, Brazil. The other 6 participants were considered "novices," having performed a maximum of 50 diagnostic cerebral angiographies before being exposed to this training model. Two of these individuals were in the neurointerventional fellowship program, and 4 were in the interventional cardiology program at the Federal University of Minas Gerais Hospital das Clínicas.

\section{Procedure Simulation Exercises}

\section{Simulation of Cerebral Angiography}

After the HPs were obtained from the pathology department, the vessels were cleaned with an injection of normal saline under pressure in the 2 arteries and 1 vein through the umbilical cord. This procedure was described by the first author (M.M.R.d.O.) and colleagues in a previous publication. ${ }^{12}$ The HP was placed in the "head" position of the endovascular unit table. A 6-Fr Foley catheter was placed in a placental artery and tied in place with a
No. 0 silk suture. A 90-cm guiding catheter (Guider Soft Tip) was connected to the HP to simulate the operator's position in a femoral approach. The guiding catheter was connected to an intravenous line with normal saline so that blood flow could be imitated by continuous infusion via a pump (Figs. 1 and 2). HP angiography was performed by hand injection of contrast medium.

\section{Stent Placement}

Following the same steps as described above for HP angiography, a road-mapping image was created. A Rebar 18 microcatheter was advanced over a 0.014-inch Transend microguidewire through the placental vessels, until the desired position was reached. Then, a Solitaire $4 \times 15-\mathrm{mm}$ stent was deployed inside the placental vessel in a standard fashion. Nonsubtracted angiography was performed to visualize the stent position inside the vessel (Fig. 3).

\section{Coil Embolization of an Aneurysm}

The HP was prepared as in the first exercise described above in Simulation of Cerebral Angiography. Angiography was performed to localize the previously created aneurysm (Fig. 4). Under road mapping, an Excelsior SL10 microcatheter was advanced over a 0.014-inch Transend microguidewire through the placental arteries and was placed inside the aneurysm sac. Then, 2 GDCs were deployed to occlude the aneurysm (Figs. 5 and 6).

\section{Onyx Injection Into a Previously Cut HP Artery}

An HP artery was cut using a No. 11 blade. Angiogra-

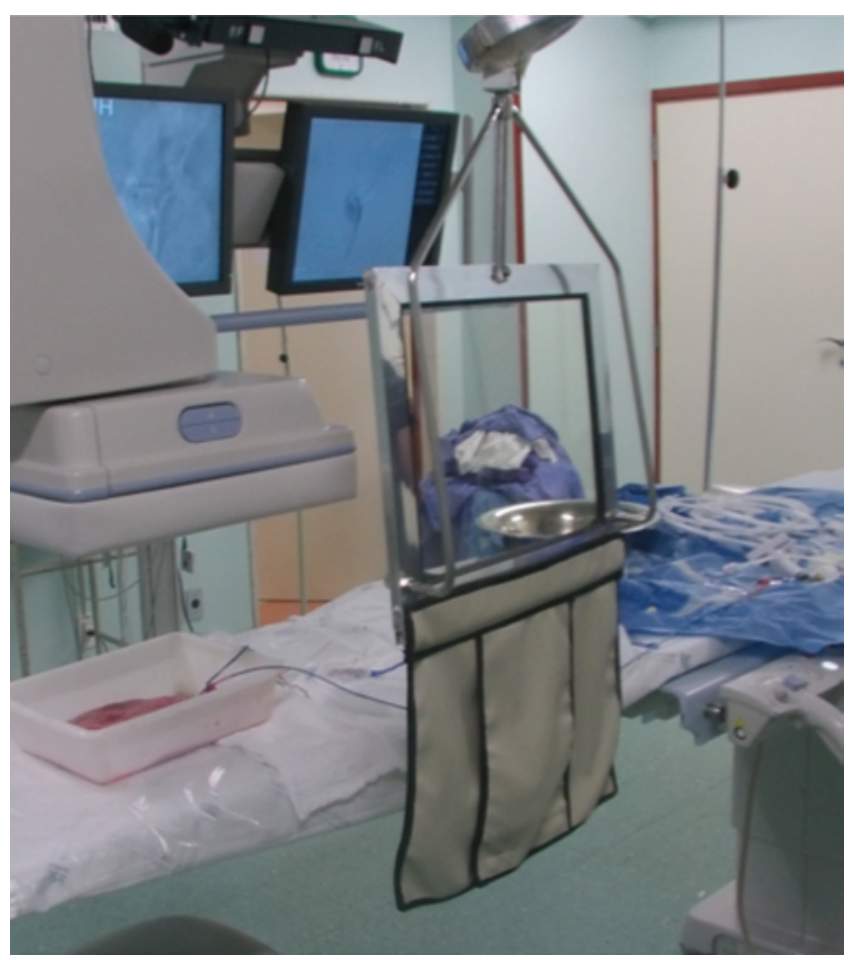

FIG. 1. Photograph showing placement of the HP in the angiography suite. The HP is in the "head" position with the guiding catheter simulating the distance between the femoral artery and the carotid artery. Figure is available in color online only. 


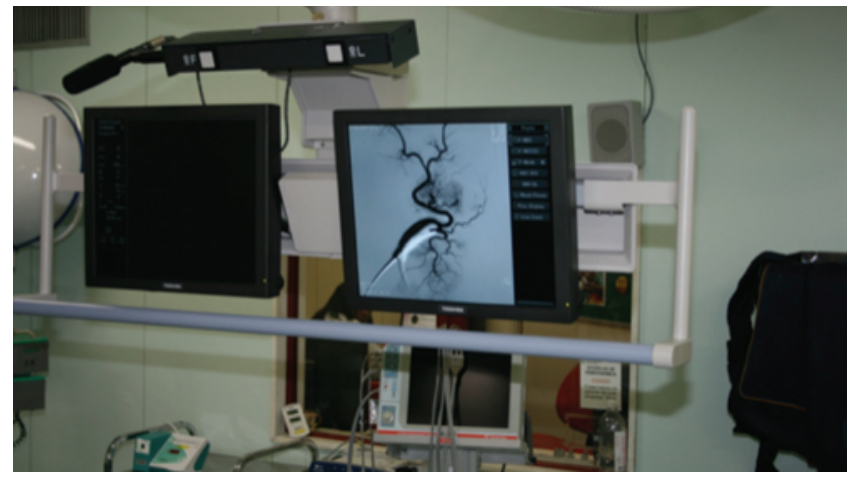

FIG. 2. Photograph showing placental angiography in process. Figure is available in color online only.

phy performed after the intentional vessel injury showed extravasation of contrast medium. A Marathon microcatheter was advanced over a 0.008 -inch Mirage microguidewire through the placental arteries until it reached the injured vessel. The microcatheter was flushed with dimethyl sulfoxide (DMSO) and the dead space was filled with Onyx-18 for 40 seconds. Under continuous subtraction fluoroscopy, intermittent very slow Onyx injections were performed, filling the placental vessels (Fig. 7).

\section{Results}

All of the above exercises were performed with the aim of reproducing real patient situations with haptic feedback. These models offered a variety of training scenarios for both novice and experienced neurointerventionalists.

All physicians in the trial answered a questionnaire using a 5-point Likert-type scale to evaluate face and content validity. Construct validity was evaluated by measuring the average time to complete all proposed simulated tasks in a single HP. The results of face, content, and construct validity assessment are summarized in Tables $1-3$, respectively.

\section{Discussion}

The HP model may be employed as a useful simulator for neurointerventional training. This model has the potential to simulate many of the important aspects of neurointerventional procedures except for initial arterial access. One could, however, potentially incorporate silicone models to simulate the arterial puncture and proximal catheter navigation to create a more realistic training model. ${ }^{13}$

The main advantages of the HP model, as shown in Table 4, are its relatively low costs, minimal infrastructure requirements, and ease of preparation and setup without the ethical concerns of animal models. A single HP can be used for multiple simulation targets and can reproduce various endovascular anatomical situations, allowing for expedited device development, modification, and testing. The absence of pulsation flow simulation in the HP model could be overcome by the use of a programmable pulsatile infusion pump, generating variable flow pressures and volumes at predetermined intervals. The lack of great vessel

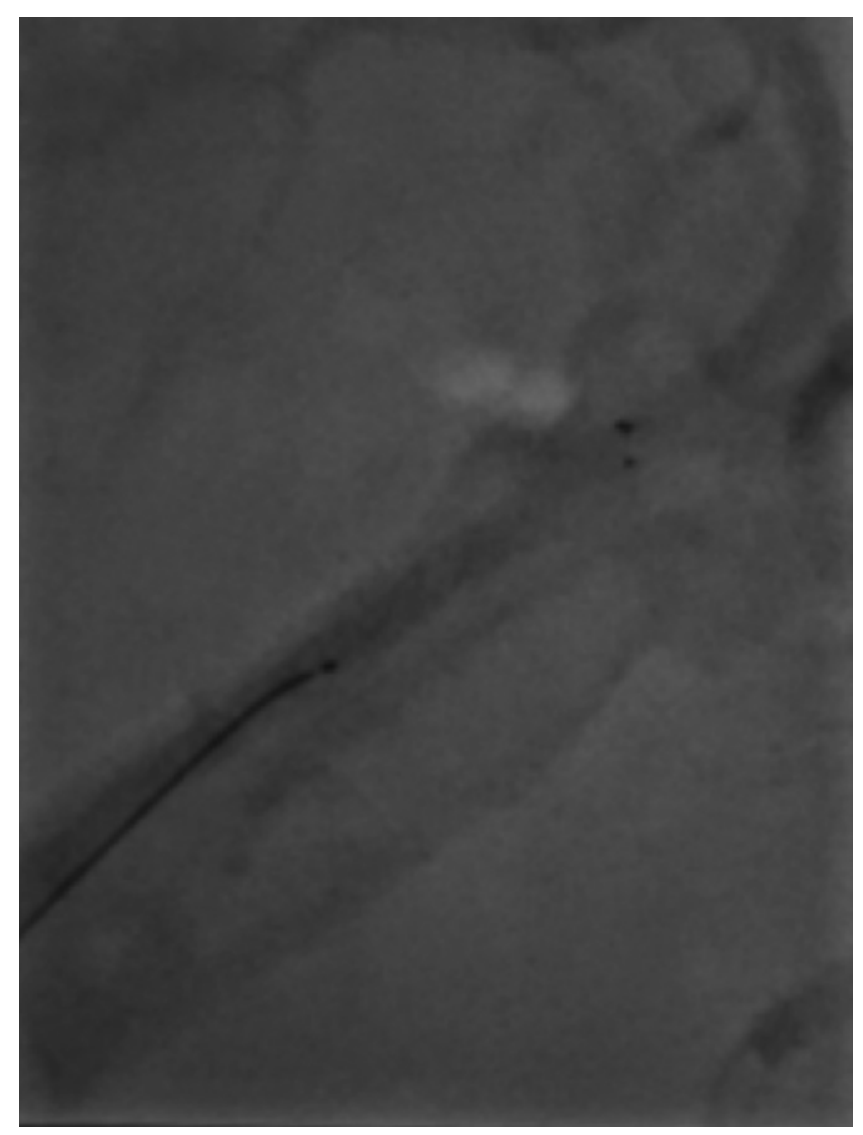

FIG. 3. Angiogram showing placement of a stent in a placental vessel.

and arch navigation could be modeled by incorporating a mannequin with a silicon simulation of the aortic arch and great vessels attached to the HP. The disadvantages of the HP model are the potential for biological contamination and the necessity of developing a multidisciplinary support structure with the departments of obstetrics and pathology.

Computer-based virtual models are now readily available as training tools for interventional radiology. Their main advantage is that they do not require any biological tissues/specimens, while their main disadvantage is their

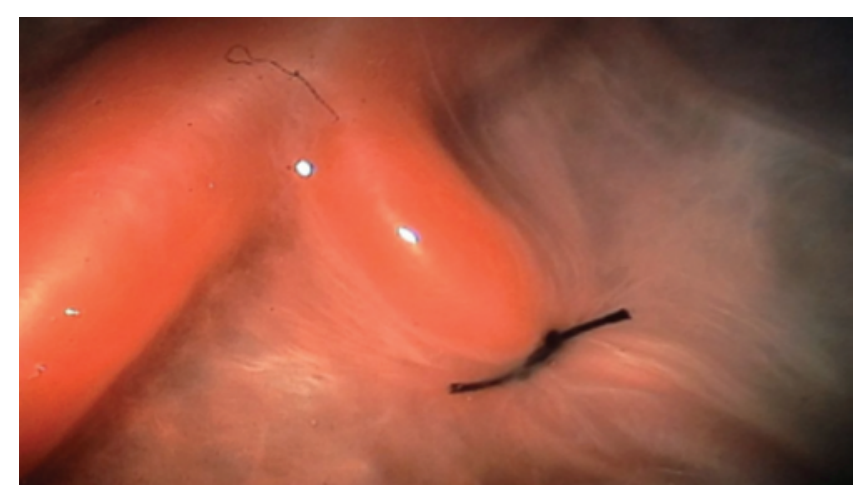

FIG. 4. Photograph of a placental aneurysm. Figure is available in color online only. 

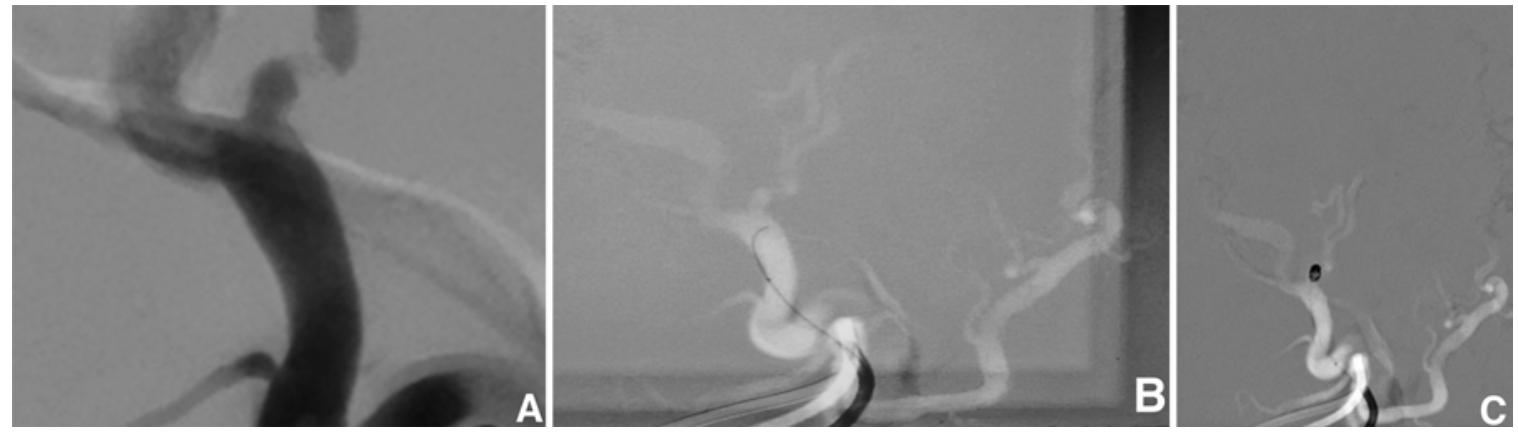

FIG. 5. Coil embolization of the placental aneurysm. A: Angiogram showing the small-neck aneurysm that was created in the placenta. B: Catheter navigation toward the aneurysm using road map views. C: Coil embolization of the aneurysm.

cost, limiting their availability in many centers. ${ }^{8,10,11}$ Some of the advanced systems can be programmed to simulate human vascular anatomy and reproduce cerebrovascular pathology. Reproducing the haptics or feel of the procedure is more difficult with these systems, and this is one aspect of the HP model that needs more study. It is possible to employ both types of these model systems in training, utilizing the HP model to develop psychomotor tactile memory to enhance the more realistic development of the virtual reality simulators. ${ }^{2,4,6}$

Animal models remain very important for training and therapeutic development throughout medicine. One of their greatest advantages lies in the ability for longitudinal follow-up studies in vivo, such as in cerebrovascular stent research. However, they require significant infrastructure, ethics and animal welfare committee oversight, and highly trained professionals to maintain the laboratories and to reproduce cerebrovascular pathology. ${ }^{5}$ These facilities and resources are generally limited to large, tertiary academic medical centers. ${ }^{8}$

A single HP model used in endovascular training can be recycled for microsurgical training, as previously described..$^{12}$ This maximizes the educational benefits from a single donor.

Although we used normal saline in our initial studies, it is possible to infuse any liquid and/or blood products into the HP model to examine such features as the acute coagulation cascade reactions during the placement of stents and related devices. The model may also be used to examine flow dynamics in both untreated and treated cerebrovascular pathology. However, a significant limitation is the inability to use the HP model for long-term studies

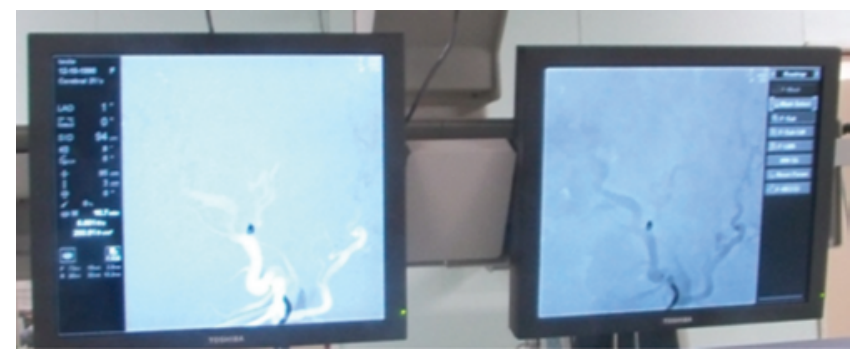

FIG. 6. Posttreatment angiogram and road map view demonstrating occlusion of the aneurysm. Figure is available in color online only. examining the pathological interaction of implants with vascular structures.

While this paper was under review, a report on the use of the HP in interventional neuroradiology was issued. ${ }^{9}$ The focus in that report was the use of HP in endovascular research, especially with the use of the Pipeline Embolization Device (ev3/Covidien). The authors briefly commented that many endovascular procedures could be performed in the placenta but did not report its use as a simulator. Our description is a detailed guide to the most common interventional procedures performed on HP, focusing on its use as a simulation model.

Face validity is the similarity of the simulator to what
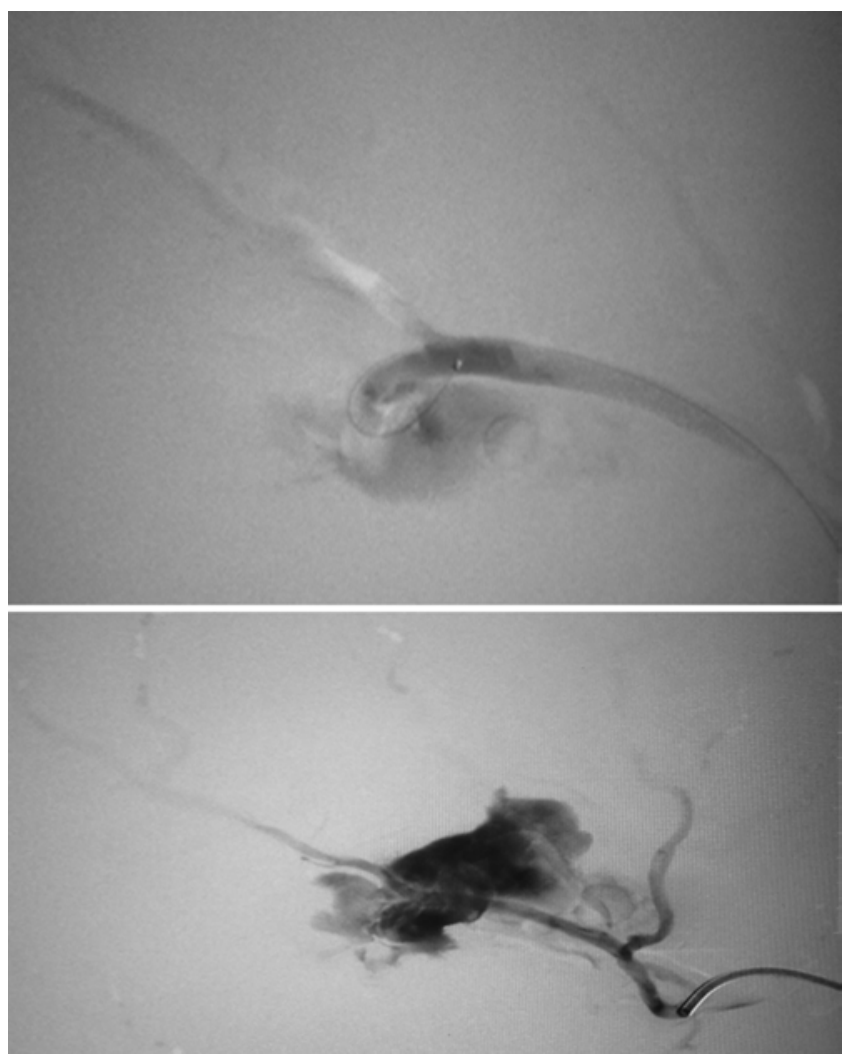

FIG. 7. Onyx injection into an injured placental vessel. Upper: Earlyphase angiogram. Lower: Late-phase angiogram. 
TABLE 1. Face validity*

\begin{tabular}{lcc}
\hline & \multicolumn{2}{c}{ Question and Response } \\
\cline { 2 - 3 } & $\begin{array}{c}\text { Can HP simulate the most } \\
\text { commonly performed } \\
\text { interventional neuroradiology } \\
\text { procedures? }\end{array}$ & $\begin{array}{c}\text { Can HP simulate } \\
\text { interventional radiology } \\
\text { patient room setup? }\end{array}$ \\
\hline Staff 1 & 4 & 3 \\
\hline Staff 2 & 4 & 3 \\
\hline Staff 3 & 4 & 3 \\
\hline Staff 4 & 4 & 3 \\
\hline Staff 5 & 4 & 3 \\
\hline Staff 6 & 4 & 3 \\
\hline Novice 1 & 4 & 3 \\
\hline Novice 2 & 4 & 3 \\
\hline Novice 3 & 4 & 3 \\
\hline Novice 4 & 4 & 3 \\
\hline Novice 5 & 4 & 3 \\
\hline Novice 6 & 4 & 3 \\
\hline Overall mean & 4 & 3 \\
\hline
\end{tabular}

* Responses were based on a 5-point Likert-type scale in which 5 indicated exactly like, 4 very similar, 3 reasonably similar, 2 poorly similar, and 1 not similar.

one wants to simulate. ${ }^{13}$ Because of the biological characteristics of HP, working with this model was found by all physicians participating in the study to provide a similar experience to real interventional neuroradiology procedures. The great advantage of the HP model was simulation of the end target task, or the final step in an interventional neuroradiology procedure. Patient setup in the endovascular theater was found to be reasonably similar when using the HP model. Patient contact, anesthesia, and sterile draping were the most important factors that caused the doctors to say that the model was lacking in similarity to real situations.

Content validity is the capacity of the simulator to perform all steps usually performed in the clinical setting. ${ }^{13}$ HP satisfies this validation from biological contamination precautions needed in the setup of the exercises to the final haptic maneuver necessary in each diagnostic or therapeutic simulated step. Femoral artery puncture was not validated by this simulator, which offers no training for this task. Catheter navigation simulation was found to be very similar, but not exactly similar due to the lack of tortuosity and obstructions in the intravenous line simulating the aorta navigation. Angiographic images were evaluated as exactly like clinical images, and this is due to the biological properties of this simulation. Experts found the device maneuvers executed to treat a lesion in the simulation to be exactly like as in the clinical setting. The novices had only carried out diagnostic angiography, so they were unable to compare and/or evaluate the HP endovascular techniques to therapeutic procedures performed in patients.

Construct validity measures the statistical difference between experts and novices regarding time to execute the whole task. ${ }^{1}$ Due to the similar biological properties of the model and the brain vessels, there was a difference in time to the execution of the predetermined procedure when comparing the 2 groups in the majority of tasks. There was no statistically significant difference between the 2 groups with respect to diagnostic angiography because the novices already had experience with this procedure from working with the simulator.

TABLE 2. Content validity*

\begin{tabular}{|c|c|c|c|c|c|}
\hline \multirow[b]{2}{*}{ Participant } & \multicolumn{5}{|c|}{ Statement and Level of Agreement } \\
\hline & $\begin{array}{l}\text { HP simulates biological } \\
\text { contamination precautions } \\
\text { in the endovascular theater. }\end{array}$ & $\begin{array}{l}\text { HP simulates } \\
\text { femoral artery } \\
\text { puncture. }\end{array}$ & $\begin{array}{c}\text { HP simulates } \\
\text { endovascular } \\
\text { catheter navigation. }\end{array}$ & $\begin{array}{c}\text { HP simulates } \\
\text { angiographic } \\
\text { imaging visualization. }\end{array}$ & $\begin{array}{c}\text { HP simulates diagnostic } \\
\text { and therapeutic haptic } \\
\text { device maneuvers. }\end{array}$ \\
\hline Staff 1 & 5 & 1 & 4 & 5 & 5 \\
\hline Staff 2 & 5 & 1 & 4 & 5 & 5 \\
\hline Staff 3 & 5 & 1 & 4 & 5 & 5 \\
\hline Staff 4 & 5 & 1 & 4 & 5 & 5 \\
\hline Staff 5 & 5 & 1 & 4 & 5 & 4 \\
\hline Staff 6 & 5 & 1 & 4 & 5 & 5 \\
\hline Mean & 5 & 1 & 4 & 5 & 5 \\
\hline Novice 1 & 5 & 1 & 4 & 5 & $5 \dagger$ \\
\hline Novice 2 & 5 & 1 & 5 & 5 & $5 \dagger$ \\
\hline Novice 3 & 5 & 1 & 4 & 5 & $5 \dagger$ \\
\hline Novice 4 & 5 & 1 & 4 & 5 & $5 \dagger$ \\
\hline Novice 5 & 5 & 1 & 4 & 5 & $5 \dagger$ \\
\hline Novice 6 & 5 & 1 & 4 & 5 & $5 \dagger$ \\
\hline Mean & 5 & 1 & 4 & 5 & $5 \dagger$ \\
\hline
\end{tabular}


TABLE 3. Construct validity*

\begin{tabular}{lcccc}
\hline \multicolumn{1}{c}{ Participant } & $\begin{array}{c}\text { Time Necessary for HP } \\
\text { Angiography }\end{array}$ & $\begin{array}{c}\text { Time Necessary to Complete } \\
\text { Aneurysm Obliteration }\end{array}$ & $\begin{array}{c}\text { Time Necessary to } \\
\text { Place Stent }\end{array}$ & $\begin{array}{c}\text { Time Necessary to Inject Onyx } \\
\text { in Predetermined Vessel }\end{array}$ \\
\hline Staff 1 & 7 & 17 & 14 & 7 \\
\hline Staff 2 & 8 & 16 & 14 & 7 \\
\hline Staff 3 & 9 & 18 & 15 & 8 \\
\hline Staff 4 & 9 & 18 & 13 & 7 \\
\hline Staff 5 & 7 & 16 & 17 & 8 \\
\hline Staff 6 & 8 & 17 & 15 & 8 \\
\hline \multicolumn{1}{c}{ Median (range) } & $8(7-9)$ & $17(16-18)$ & $14.5(13-17)$ & $7.5(7-8)$ \\
\hline Novice 1 & 13 & 49 & 37 & 18 \\
\hline Novice 2 & 12 & 51 & 35 & 19 \\
\hline Novice 3 & 14 & 49 & 39 & 19 \\
\hline Novice 4 & 14 & 51 & 35 & 18 \\
\hline Novice 5 & 14 & 48 & 36 & 20 \\
\hline Novice 6 & 13 & 50 & 38 & 21 \\
\hline \multicolumn{1}{c}{ Median (range) } & $13.5(12-14)$ & $49.5(48-51)$ & $36.5(35-39)$ & $19(18-21)$ \\
\hline p Value† & 0.003 & 0.004 & 0.004 & 0.003 \\
\hline
\end{tabular}

${ }^{*}$ All data are in minutes.

$\dagger$ Mann-Whitney test.

TABLE 4. Advantages and disadvantages of neurointerventional simulators

\begin{tabular}{llllll}
\hline \multicolumn{1}{c}{ Model } & Costs & Infrastructure Needed & Haptics & Human Vascular Anatomy & Real Patient Technical Similarity \\
\hline Animal & High & Large & Good & No & Yes \\
\hline Cadaver & High & Large & Midrange & Yes & Midrange \\
\hline Virtual & High & Small & Poor & Reproducible & Poor \\
\hline Placenta & Low & Small & Good & No & Yes \\
\hline Silicon & Medium & Small & Midrange & Reproducible & Midrange \\
\hline
\end{tabular}

We do not expect to be able to draw any strong conclusions about this methodology based on results from only one center, and a multicenter study is needed.

We believe that one of the main benefits of the HP model is the reproduction of haptics similar to actual endovascular treatments. Although the development of tactile feedback in virtual models will improve with technological advances, high costs will continue to limit the availability of virtual models.

\section{Conclusions}

Human placenta can be a useful training model in neurointerventional surgery due to the excellent haptics, low startup costs, and ready availability for any institution with interventional capabilities. Assessment of face, content, and construct validity in a single center confirms the educational benefits of this model, but multicenter validation is necessary for definitive conclusions regarding predictive validation.

\section{References}

1. Abboudi H, Khan MS, Aboumarzouk O, Guru KA, Chal- lacombe B, Dasgupta P, et al: Current status of validation for robotic surgery simulators-a systematic review. BJU Int 111:194-205, 2013

2. Azarnoush H, Alzhrani G, Winkler-Schwartz A, Alotaibi F, Gelinas-Phaneuf N, Pazos V, et al: Neurosurgical virtual reality simulation metrics to assess psychomotor skills during brain tumor resection. Int J Comput Assist Radiol Surg 10:603-618, 2015

3. Bath J, Lawrence P: Why we need open simulation to train surgeons in an era of work-hour restrictions. Vascular 19:175-177, 2011

4. Choudhury N, Gélinas-Phaneuf N, Delorme S, Del Maestro R: Fundamentals of neurosurgery: virtual reality tasks for training and evaluation of technical skills. World Neurosurg 80:e9-e19, 2013

5. Corbett TJ, Doyle BJ, Callanan A, Walsh MT, McGloughlin TM: Engineering silicone rubbers for in vitro studies: creating AAA models and ILT analogues with physiological properties. J Biomech Eng 132:011008, 2010

6. Delorme S, Laroche D, DiRaddo R, Del Maestro RF: NeuroTouch: a physics-based virtual simulator for cranial microneurosurgery training. Neurosurgery 71 (1 Suppl Operative):32-42, 2012

7. Fearing NM: The use of simulation in medical and surgical education. Mo Med 110:123-124, 2013

8. Kobayashi E, Hishikawa S, Teratani T, Lefor AT: The pig as a model for translational research: overview of porcine ani- 
mal models at Jichi Medical University. Transplant Res 1:8, 2012

9. Kwok JC, Huang W, Leung WC, Chan SK, Chan KY, Leung $\mathrm{KM}$, et al: Human placenta as an ex vivo vascular model for neurointerventional research. J Neurointerv Surg 6:394399, 2014

10. Marcus H, Vakharia V, Kirkman MA, Murphy M, Nandi D: Practice makes perfect? The role of simulation-based deliberate practice and script-based mental rehearsal in the acquisition and maintenance of operative neurosurgical skills. Neurosurgery 72 (Suppl 1):124-130, 2013

11. Mitha AP, Almekhlafi MA, Janjua MJ, Albuquerque FC, McDougall CG: Simulation and augmented reality in endovascular neurosurgery: lessons from aviation. Neurosurgery 72 (Suppl 1):107-114, 2013

12. Oliveira Magaldi M, Nicolato A, Godinho JV, Santos M, Prosdocimi A, Malheiros JA: Human placenta aneurysm model for training neurosurgeons in vascular microsurgery. Neurosurgery 10 (Suppl 4):592-601, 2014

13. Sinha S, Cooling N: Simulation based education-models for teaching surgical skills in general practice. Aust Fam Physician 41:985-988, 2012

\section{Disclosure}

The authors report no conflict of interest concerning the materi- als or methods used in this study or the findings specified in this paper.

\section{Author Contributions}

Conception and design: Ribeiro de Oliveira, Santos, Prosdocimi, Sabbagh, Reis, Del Maestro. Acquisition of data: Ribeiro de Oliveira, Santos, Godinho, Martins, Prosdocimi, Reis. Analysis and interpretation of data: Ribeiro de Oliveira, Godinho,

Martins, Reis. Drafting the article: Ribeiro de Oliveira, Trivelato. Critically revising the article: Ribeiro de Oliveira, Nicolato, Godinho, Prosdocimi, Trivelato, Del Maestro. Reviewed submitted version of manuscript: Ribeiro de Oliveira, Nicolato, Brito, Alvarenga, Martins, Sabbagh, Reis. Approved the final version of the manuscript on behalf of all authors: Ribeiro de Oliveira. Statistical analysis: Ribeiro de Oliveira. Administrative/technical/ material support: Ribeiro de Oliveira, Prosdocimi. Study supervision: Ribeiro de Oliveira, Nicolato, Brito, Alvarenga, Sabbagh, Reis.

\section{Correspondence}

Marcelo Magaldi Ribeiro de Oliveira, Rua Montes Claros 1442101, Belo Horizonte, Minas Gerais 30310-370, Brazil. email: mmagaldi@hotmail.com. 